\title{
Melanom: Wer profitiert von Interferon?
}

\author{
Der Wert von Interferon $a-2 b$ (IFN) bei Patienten mit malignem Melanom ist \\ umstritten. Zumindest eine Subgruppe scheint aber von dem Zytokin zu \\ profitieren. Welche das ist, zeigt sich in einer Langzeitanalyse.
}

nterferon war einst Hoffnungsträger, doch die Erfolge einer adjuvanten Therapie beim Melanom sind im besten Falle als mäßig zu bezeichnen. Nur eine Minderheit der Patienten scheint von der Therapie zu profitieren. Welche Patienten das sind, wird seit Langem untersucht - u.a. in zwei großen EORTC-Studien. Von einer dieser Studien liegt nun ein Langzeit-Follow-up vor. Behandelt wurden 1.388 Patienten mit einem Melanom im Stadium IIB/III, das reseziert wurde. Sie erhielten 4 Wochen lang 5-mal pro Woche 10 MU Interferon, gefolgt von entweder 3-mal wöchentlich 10 MU für 12 Monate (IFN 13) oder 3-mal wöchentlich $5 \mathrm{MU}$ für 24 Monate (IFN 25); eine Kontrollgruppe wurde lediglich beobachtet.
Nach einem medianen Follow-up von 11 Jahren ergaben sich für IFN 13 bzw. für IFN 25 im Vergleich zur Kontrollgruppe folgende Hazard Ratios (HR): —rezidivfreies Überleben (RFS): 0,94

$(\mathrm{p}=0,46)$ versus $0,84(\mathrm{p}=0,06)$

_ fernmetastasenfreies Überleben

(DMFS): 0,95 ( $\mathrm{p}=0,57)$ versus 0,84

$(\mathrm{p}=0,07)$

_Gesamtüberleben (OS): 0,95

$(\mathrm{p}=0,58)$ versus $0,84(\mathrm{p}=0,08)$

Der Einfluss der Behandlung war für Patienten mit ulzerierten Melanomen am größten, bei den übrigen null $(\mathrm{HR} \geq 1,0)$.

So ergaben sich für IFN 13 bzw. IFN 25 gegenüber der Kontrollbehandlung bei Patienten mit ulzeriertem Melanom folgende HR:

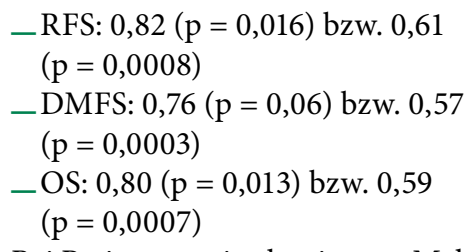

Bei Patienten mit ulzeriertem Melanom im Stadium IIB/III-N1 (nur mikroskopischer Lymphknotenbefall) ergaben sich folgende geschätzte HR:

-RFS: 0,85 bzw. 0,62

_DMFS: 0,80 bzw. 0,56

_OS: 0,77 bzw. 0,54

Fazit: Diesen Ergebnissen zufolge ist eine 25-monatige Gabe von IFN der nur 13-monatigen Gabe in höherer Dosierung bei Patienten mit kutanem Melanom überlegen, und zwar v. a. bei ulzerierten Melanomen.

Christina Berndt

Eggermont AMM et al. Long term follow up of the EORTC 18952 trial of adjuvant therapy in resected stage IIB-III cutaneous melanoma patients comparing intermediate doses of interferon-alpha-2b (IFN) with observation: Ulceration of primary is key determinant for IFN-sensitivity. Eur J Cancer. 2016;55:111-21.

\section{Melanom: Wächterlymphknoten auf Prüfstand}

\author{
Ein von Tumorzellen befallener Wächterlymphknoten gilt als Warnsignal. Seit \\ einigen Jahren werden bei einem solchen Befund sämtliche Lymphknoten \\ ausgeräumt. Aber wirkt sich das auf die Überlebenschancen der Patienten aus?
}

D atienten mit positivem Wächterlymphknoten wird üblicherweise eine vollständige Lymphknoten-Dissektion (LND) empfohlen, deren Effekt auf den Krankheitsverlauf allerdings bis heute kontrovers diskutiert wird. In der deutschen Phase-III-Studie DeCOGSLT wurde deshalb untersucht, ob die radikale LND im Vergleich zur Beobachtung das Überleben verlängert. Patienten mit kutanem Melanom und positivem Wächterlymphknoten erhielten randomisiert entweder eine vollständige LND oder wurden nur beobachtet. Dabei wurden sie nach Dicke ( $>$ oder $<2 \mathrm{~mm}$ ) und Ulzeration des Primärtumors sowie einer intendierten adjuvanten Interferontherapie stratifiziert. Primärer Endpunkt war das fernmetastasenfreie Überleben.
Bei 1.269 von 5.547 Patienten (23\%) hatte die Biopsie des Wächterlymphknotens Mikrometastasen ergeben. Von ihnen willigten nur 483 (39\%) in die Teilnahme an der Studie ein, weshalb diese vorzeitig geschlossen wurde. Analysiert wurden letztlich 233 mit LND bzw. 240 Patienten ohne. Zudem erfolgte eine Subgruppenanalyse jener 311 Patienten (66\%), die Metastasen in den Wächterlymphknoten von $\leq 1 \mathrm{~mm}$ Durchmesser gezeigt hatten; von ihnen befanden sich 158 in der Beobachtungs- und 153 in der Interventionsgruppe.

Nach 35 Monaten lag das fernmetastasenfreie 3-Jahres-Überleben bei 77,0 \% in der Beobachtungs- und bei $74,9 \%$ in der Interventionsgruppe. Für die Subgruppe mit Metastasen $\leq 1 \mathrm{~mm}$ betrug der Anteil der fernmetastasenfreien
Überlebenden nach 3 Jahren 80,5\% in der Beobachtungs- und 79,5\% in der Interventionsgruppe.

In der Interventionsgruppe traten Nebenwirkungen vom Schweregrad 3 bzw. 4 bei 15 (6\%) bzw. 19 (8\%) Patienten auf, darunter Lymphödeme und -fisteln, Serome, Infektionen und verzögerte Wundheilung.

Fazit: Wegen des vorzeitigen Abbruchs der Studie ist diese statistisch nur bedingt belastbar. Dennoch zeigen die Daten, dass es keinen Unterschied mit Blick auf das fernmetastasenfreie Überleben macht, ob Melanompatienten mit positiven Wächterlymphknoten eine vollständige LND erhalten oder nicht. Eine solche Intervention sollte demnach zumindest dann nicht empfohlen werden, wenn die Metastasen einen Durchmesser von $\leq 1 \mathrm{~mm}$ haben. Christina Berndt

Leiter $U$ et al. Complete lymph node dissection versus no dissection in patients with sentinel lymph node biopsy positive melanoma (DeCOG-SLT): a multicentre, randomised, phase 3 trial. Lancet Oncol. 2016;17(6):757-67. 